\title{
Development of a Dynamic Environment Transmission Electron Microscope for the Study of Fast Phenomena in Nanoscale Materials
}

\author{
Renske M. van der Veen ${ }^{1 *}$ \\ 1. University of Illinois at Urbana-Champaign, Department of Chemistry, Urbana, USA. \\ * Corresponding author: renske@illinois.edu
}

In situ transmission electron microscopy has become a powerful technique to study functional nanomaterial dynamics in response to electrical, chemical, electrochemical, thermal, and/or electromagnetic fields prevalent under operating conditions. The time resolution, however, is typically limited by the maximum frame rate of the detector, which is at best in the $\mathrm{kHz}$ regime. Ultrafast transmission electron microscopy combines the high time resolution of laser spectroscopy with the excellent spatial resolution of electron microscopy techniques. The structural and electronic changes in the material are initiated by short (fs, ps, ns) laser pulses, which are followed by similarly short photoelectron pulses for probing the dynamics by means of imaging, diffraction, or energy-loss spectroscopy (EELS) within the electron microscope. In this talk I will present our dynamic environment transmission electron microscope (DETEM) at the University of Illinois (Fig. 1). This versatile instrument enables time-resolved in situ studies of fast (fs-ms) material processes in gaseous and liquid environments that are triggered by light, heat, chemical flux, electrical bias or strain.

The setup is based on a 100-300 keV Hitachi H-9500 TEM, equipped with a thermionic $\mathrm{LaB}_{6}$ gun, and a Gatan Imaging Filter (GIF) Quantum ER with Gatan K2 IS direct electron detection camera. The microscope is interfaced with a fs laser system (Light Conversion Pharos with Orpheus-F), that allows excitation of the sample with wavelengths between $210-2600 \mathrm{~nm}$ and variable repetition rates up to 1 MHz, as well as two ns lasers (Spectra Physics VGEN HE and Bright Solutions Wedge HF). Various modes of operation are implemented in order to span a broad range of time scales: fs and ns stroboscopy for reversible dynamics (300 fs-1 ns), ns-resolved single-shot probing for irreversible dynamics ( $20 \mathrm{~ns})$, as well as low-repetition rate $(400 \mathrm{~Hz})$ pump-probe microscopy using the $K 2 I S$ camera synchronized to the pulsed electron beam. The latter mode should allow us to circumvent the "rolling-shutter" problem commonly encountered in fast microscopies. The unique in situ capabilities of the DETEM instrument (gas exposure, liquids and high temperatures), in combination with electron-energy loss spectroscopy (EELS) and sensitive direct-electron detection, will enable transformative studies in the fields of photocatalysis, field-induced nanoparticle assembly, and photoinduced phase transitions. I will give examples of the first studies performed with this tool, including photoinduced spin-state switching in metalorganic materials and liquid-phase experiments. 

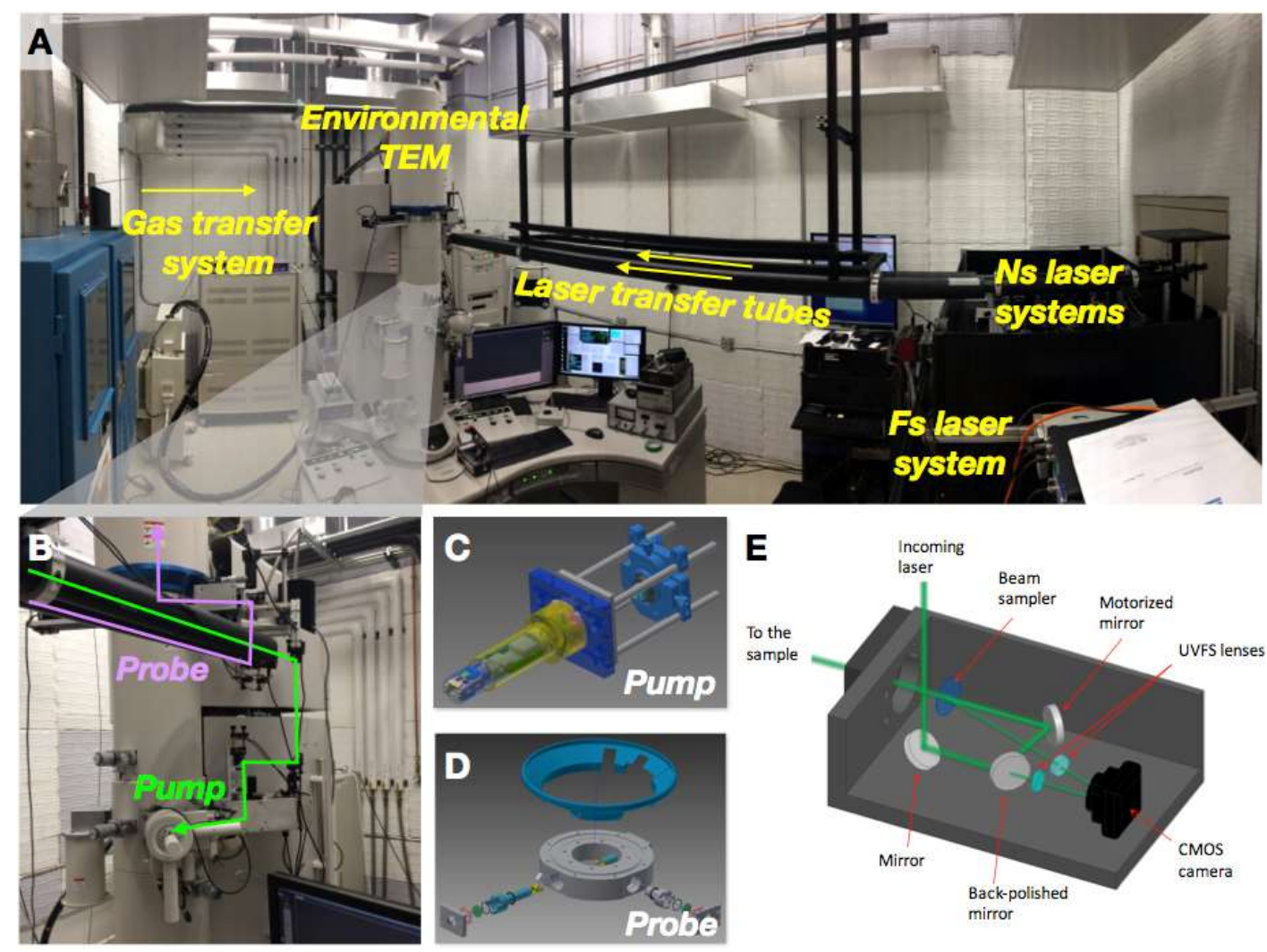

Figure 1. A. Overview of the DETEM setup at the MRL developed in the van der Veen lab; B. pump and probe laser beam paths into the microscope; C. probe laser insert; D. pump laser insert; E. pump laser box that allows on-the-fly beam tracking and pointing stabilization. 